\title{
Nano Crystalline Titania Powders as Enhancement for 2-(2-Nitrobenzylidene) Hydrazinecarbothioamide as Corrosion Inhibitor
}

\author{
Zeyad Fadhil \\ College of Pharmacy, Thi-Qar University, Thi-Qar-Iraq.
}

\begin{abstract}
The present investigation, represent arranging of nano-sized $\mathrm{TiO}_{2}$ powder by method for sol-gel strategy through precursor hydrolysis of Titanium isopropoxide. Morphological reviews gained from SEM micrograph showed the particles with the round shapes are Anatase in nature. Crystalline size of $\mathrm{TiO}_{2}$ powder has procured is $50-75 \mathrm{~nm}$ for Anatase at $550{ }^{\circ} \mathrm{C}$ by controlling the sharpness. Weight lessening system has been used to consider the disintegration prevention effectiveness of 2-(2-nitrobenzylidene) hydrazinecarbothioamide enhanced by $\mathrm{TiO}_{2}$ in $\mathrm{HCl}$ course of action. The results show that 2-(2-nitrobenzylidene) hydrazinecarbothioamide with $\mathrm{TiO}_{2}$ raise the disintegration productivity from 87 to $96 \%$. Disintegration obstacle viability increases with growing concentration of inhibitor and it moreover augments with extending joining of $\mathrm{TiO}_{2}$. [DOI: 10.22401/JNUS.20.3.01]
\end{abstract}

Keywords: $\mathrm{TiO}_{2}$, titania, Surface, Erosion.

\section{Introduction}

The fascinating of the preparation of submicron mono-scattered powders is growing. These powders find applications in the ceramic business when unrivaled materials are required. Temperatures and sintering time can be on a very basic level reduced with powders of thin molecule estimate scattering. Fine colloidal particles could be made by deferent procedures from the vapor organize or the liquid stage [1]. Lately, extraordinary impact has been focused on combination of metal oxide nano particles owing to their phenomenally assorted physical and synthetic properties concerning the mass materials. Titanium dioxide, $\mathrm{TiO}_{2}$, has been examine broadly as photocatalyst to oversee environment pollution, water purifying, wastewater treatment, unsafe waste control and air sterilization [2]. Titanium dioxide was one of most enthusiasm for creative applications as a result of its morphology and crystalline stage. $\mathrm{TiO}_{2}$ exists three various stages, i.e., Anatase, Rutile, and Brookite. The dynamic crystallite times of $\mathrm{TiO}_{2}$ are Anatase and rutile [3]. $\mathrm{TiO}_{2}$ has been for the most part considered concerning diverse applications, utilizing the photograph synergist reactant and direct conductivity, which unequivocally depend on upon the crystalline structure, morphology and crystallite estimate [3]. Sol-gel framework has been a standout among the most used systems as a result of its believability of deciding remarkable metastable structure at low reaction temperatures and heavenly mixture homogeinity [4]. Starting now, the planning of $\mathrm{TiO}_{2}$ by the sol-gel strategy has ended up being a greatly supportive to-for photograph induced molecule estimate reactions to happen on a titanium dioxide surface [5]. There are outstanding factors that impact the photo influenced reactions, including particle estimate, organize stage creation, occurrence and arranging system; for case, Anatase $\mathrm{TiO}_{2}$ nanoparticles have shown higher photocatalytic development than rutile $\mathrm{TiO}_{2}$ [6]. The use of inhibitors is one of the best methods for ensuring metals against disintegration [7]. Most utilization inhibitors are common blends having hetero particles in their fragrant or long carbon chain [8]. Regardless, there is extending stress over the danger of most disintegration inhibitors. The hazardous effect influences living structures and in addition dangerous substance the earth [9]. Generally, the characteristic blends containing hetero particles like nitrogen, oxygen and sulfur thus on have been seen to be greatly convincing disintegration inhibitors $[10,11]$. The profitability of these mixes depends on the electron-thickness of hetero particles. The deterrent capability in like manner depends on the amount of adsorption element centers in the molecule, their charge thickness, nuclear size and technique for adsorption and course of action of metallic buildings. Particles, for instance, nitrogen, 
oxygen and sulfur are fit for surrounding coordinate covalent bond with metal inferable from their free electron sets. Exacerbates that have pi-securities like aldehydes, ketones, imines similarly generally show extraordinary inhibitive properties on account of correspondence of pi-orbital with metal surface [12]. In this review the $\mathrm{TiO}_{2}$ improved for 2-(2-nitrobenzylidene) hydrazinecarbothioamide as consumption inhibitor for gentle steel in hydrochloric corrosive solution.

\section{Experimental Procedure \\ Preparation of $\mathrm{TiO}_{2}$}

$\mathrm{TiO}_{2}$ nanoparticles have been the prepared by using of titanium tetra isopropoxide as a precursor and was blended with $\mathrm{HCl}$, ethanol and deionized water blend, mixed for 60 minutes, in $\mathrm{pH}$ scope of $1.5 .10 \mathrm{ml}$ of deionized water had been added to the above blend and mixed for 2 hours at room temperature. Final the solution was dried at room temperature and the powder has been heated at $120^{\circ} \mathrm{C}$ for 60 minutes.

\section{Preparation of Schiff base}

A hot solution of ethanl $200 \mathrm{~mL}$, and thiosemicarbazide $(2.73 \mathrm{~g}, 25 \mathrm{mmol})$ a 2-nitobenzaldehyde $(25 \mathrm{mmol})$ in $200 \mathrm{~mL}$ ethanol has been added drop wise. The mix was blended and refluxed for 6 hours, it was isolated and filtrates. The item was gathered by filtration, washed with ethanol, and dried (Scheme (1)). Yellow precious stones had been formed. Yield $79 \%$, m.p. $213{ }^{\circ}$ C. Anal. IR $\left(\mathrm{KBr}, \mathrm{cm}^{-1}\right): v\left(\mathrm{NH}_{2}\right.$ and $\left.\mathrm{NH}\right) 3400.1,3383.6$ and 3266.0; $v(\mathrm{C}=\mathrm{N})$ 1611.2. 1HNMR (DMSOd6): $\delta 7.170(\mathrm{~d}, 1 \mathrm{H}) ; 7.811$ and $7.991(\mathrm{dd}, 2 \mathrm{H})$; 7.011-7.125 (m, 1H); $8.716(\mathrm{~s}, 1 \mathrm{H}, \mathrm{H}-\mathrm{C}=\mathrm{N})$; $8.77 \mathrm{~d}, 2 \mathrm{H}$ and $10.9111(\mathrm{~s}, 1 \mathrm{H})$ for $\mathrm{NH}_{2}$ and $\mathrm{NH}$ respectively.<smiles>O=Cc1ccccc1[N+](=O)[O-]</smiles><smiles>NNC(N)=S</smiles>

Hydrazinecarbothioamide<smiles>NC(=S)N/N=C\c1ccccc1[N+](=O)[O-]</smiles>

2-(2-Nitrobenzylidene)hydrazinecarbothioamide

\section{Scheme (1): Preparation of inhibitor chemically.}

\section{Weight loss Measurements}

Weight diminishment estimations were performed on rectangular mellow steel tests with the size $2.5 \times 2.0 \times 0.025 \mathrm{~cm} 3$ by soaking the gentle steel tests coupons into destructive arrangement $(100 \mathrm{~mL})$ with and without of varieties concentrations [13] of 2-(2nitrobenzylidene) hydrazinecarbothioamide with $\mathrm{TiO}_{2}$. After the elapsed by time of immersion, the coupons were taken out, washed, dried and weighed precisely. All the tests were directed in circulated air through 1 $\mathrm{M} \mathrm{HCl}$. All the analyses were performed in triplicate and normal qualities were accounted for. From the assessed weight reduction, was calculated utilizing equation (1).

$$
I E \%=\frac{w-w^{\circ}}{w} \times 100
$$

$\mathrm{w}$ and $\mathrm{w}^{\mathrm{O}}$ were the losses of weight without and/or with 2-(2nitrobenzylidene)hydrazinecarbothioamide.

\section{Results and Discussion}

Scanning Electron Microscopic (SEM) investigation. The methodologies considering electron microscopy offer purposes of enthusiasm for morphological and sizes examination; regardless, they give compelled information about the size dissemination and genuine particles normal. For SEM portrayal, nanoparticles game plan should be at first changed over into a dry powder, which is then mounted on a case holder brought after by covering with a conductive metal, for instance, $\mathrm{TiO}_{2}$, using a sputter coater. The case is then checked with a concentrated fine light 
emission [14]. The surface characteristics of the example are procured from the discretionary electrons transmitted from the case surface. The mean size got by SEM is identical with results obtained by component light scattering. Additionally, these techniques are monotonous, outlandish and infrequently require comparing information about measuring allocation [15]. $\mathrm{TiO}_{2}$ was inspected by SEM. The surface was assessed at enhancement, $50 \mathrm{k}$ x. All things considered, SEM is a particularly important technique for elucidating morphology of $\mathrm{TiO}_{2}$ and size of nanoparticles, moreover the assignment of $\mathrm{TiO}_{2}$, as showed up in Fig.(1), at enhancements of $50.00 \mathrm{k}$ x. The SEM photograph of $\mathrm{TiO}_{2}$ nanoparticles are showed up in Fig.(1). SEM picture of $\mathrm{TiO}_{2}$ nanoparticles unmistakably exhibits that in the room temperature arranged $\mathrm{TiO}_{2}$ the ordinary size of the nanoparticles is $50 \mathrm{~nm}$, with round shape.

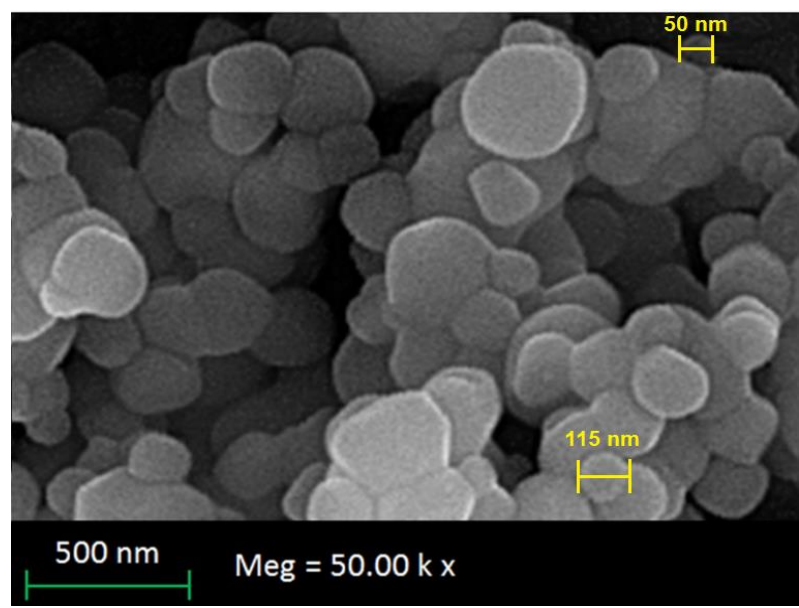

Fig.(1): SEM morphology of $\mathrm{TiO}_{2}$.

\section{Weight loss results}

The outcomes that gained for the assortment of weight decrease of mild steel with time amid the block of the disintegration of $\mathrm{HCl}$ by 2-(2nitrobenzylidene) hydrazinecarbothioamide at $303 \mathrm{~K}$ are shown in Fig.(2). From this figure, it might be seen that weight lessening of mild steel reduces with development in the grouping of the inhibitor showing that 2-(2nitrobenzylidene) hydrazinecarbothioamide is an adsorption inhibitor. For an adsorption inhibitor, weight lessening is depended upon to decrease with extension in union of the inhibitor [16].

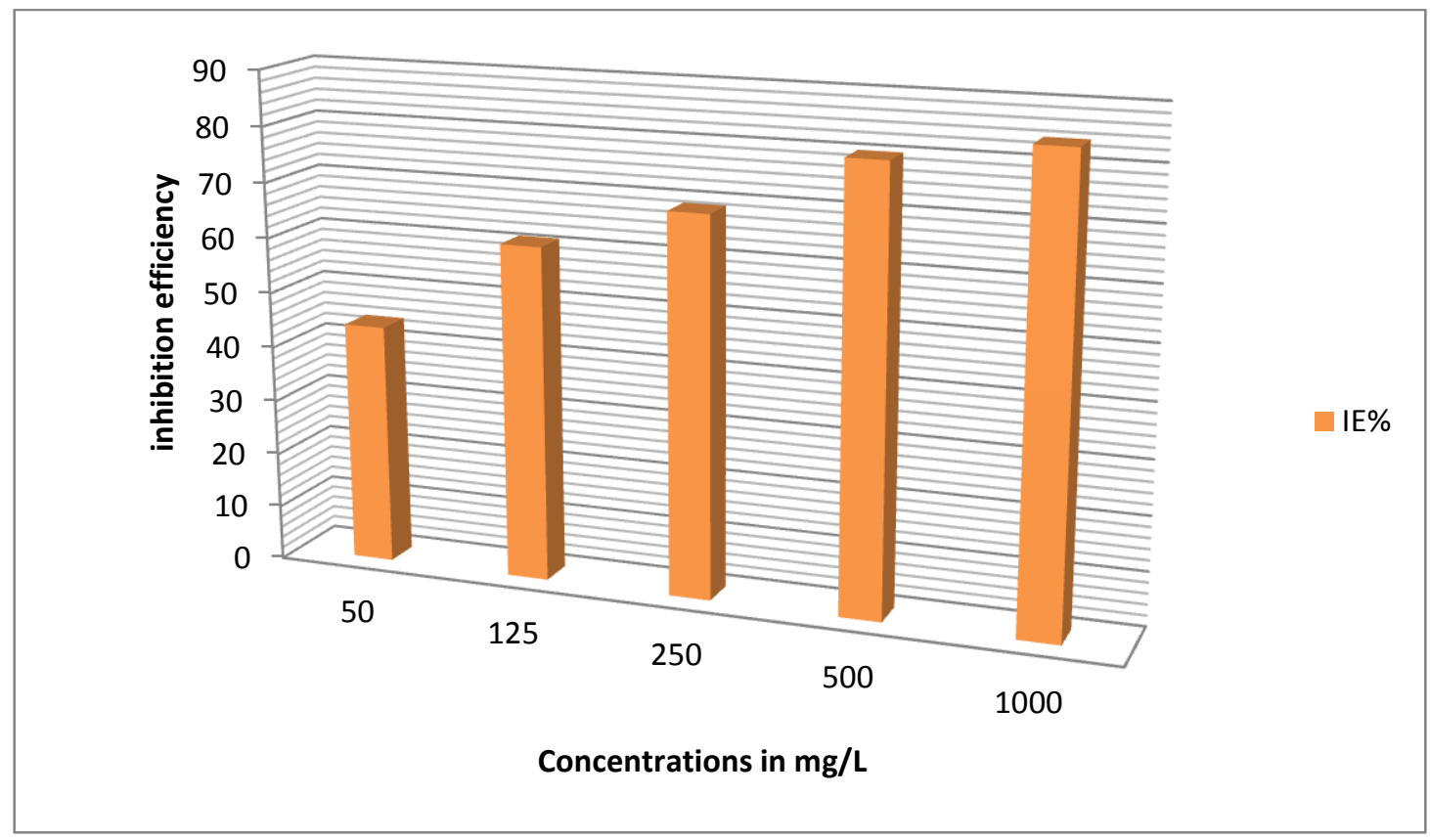

Fig.(2): Corrosion inhibition 2-(2-nitrobenzylidene) hydrazinecarbothioamide without $\mathrm{TiO}_{2}$ nanoparticales. 
Fig.(3) exhibits that utilization inhibitor at deferent concentrations improved with $50 \mathrm{mg}$ of $\mathrm{TiO}_{2}$ nanoparticales hindered the disintegration of mellow steel in $1.0 \mathrm{M} \mathrm{HCl}$ media by weight decrease estimations. It is watched that, at a $303 \mathrm{~K}$, the restraint viability extended with extension with $50 \mathrm{mg}$ of $\mathrm{TiO}_{2}$ nanoparticales. A development in limitation viability with $50 \mathrm{mg}$ of $\mathrm{TiO}_{2}$ nanoparticales demonstrates a solid communication between the $\mathrm{TiO}_{2}$ and metal surface.

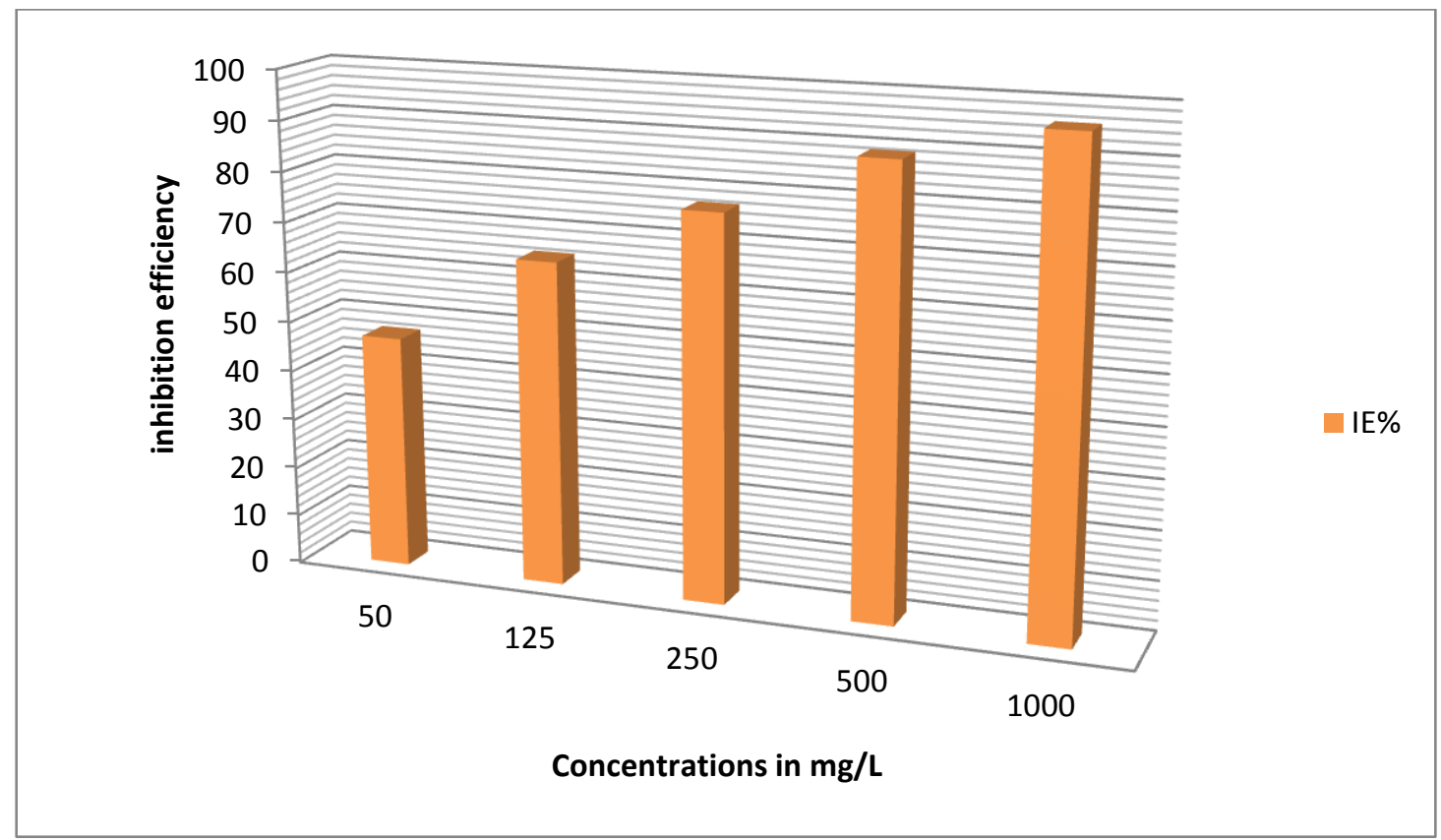

Fig.(3): Corrosion inhibition of 2-(2-nitrobenzylidene)hydrazinecarbothioamide with $50 \mathrm{mg}$ of $\mathrm{TiO}_{2}$ nanoparticales.

\section{Conclusion}

2-(2-nitrobenzylidene) hydrazinecarbothioamide go about as an inhibitor for mild steel consumption in $1 \mathrm{M} \mathrm{HCl}$ media. The restraint effectiveness expanded with increment in concentration of 2-(2-nitrobenzylidene) hydrazinecarbothioamide. Enhancement by $\mathrm{TiO}_{2}$ might be expanding the inhibition productivity.

\section{References:}

[1] Livage J., Henry M., "Jolivet. Fine particles part ii: formation mechanisms and application. MRS Bulletin" 15, 18-25, 1990.

[2] S. Perumal1, C.Gnana Sambandam 2, K. Monikanda prabu 3, S. Ananthakumar. Synthesis and Charecterization Studies Of Nano Tio2 Prepared Via Sol-Gel Method. International Journal of Research in Engineering and Technology. 3(4), (2014) 651-657).

[3] Nakano H., Hasuike H., Kisoda K., Nishio, T. Isshiki,. Harima H., "Synthesis of $\mathrm{TiO}_{2}$ nanocrystals controlled by means of the size of magnetic lements and the level of doping with them", J. Phys.: Condens. Matter, 21, 64-76, 2009.

[4] Mahshid S., Askari M., Ghamsari M., Afshar N., Lahuti S., "Mixed-phase $\mathrm{TiO}_{2}$ nanoparticles preparation using sol-gel method. Journal of Alloys and Compounds", 47, 586-589. 2009.

[5] Jang H., Kim S., Kim S., "Effect of particle size and phase composition of titanium dioxide nanoparticles on the photocatalytic properties", J. Nanopart. Res. 3, 141-147, 2001.

[6] Scaflani L., Palmisano M., "Influence of the preparation methods of titanium dioxide on the photocatalytic degradation of phenol in aqueous dispersion", J. Phys. Chem. 94, 829-832, 1990.

[7] Eddy N., Ebenso E., "Adsorption and inhibitive properties of ethanol extracts of Musa sapientum peels as a green corrosion inhibitor for mild steel in $\mathrm{H}_{2} \mathrm{SO}_{4}$ ", Afri. J. Pure Appl. Chem., 2, 46-54, 2008. 
[8] Hamdy A.,El-Gendy N.,"Thermodynamic, adsorption and electrochemical studies for corrosion inhibition of carbon steel by henna extract in acid medium, Egyptian Journal of Petroleum" 22, 17-25, 2012.

[9] Eddy N., Odoemelam S., Ogoko E., Ita E., Inhibition of the Corrosion of Zinc in 0.01 $0.04 \mathrm{M} \quad \mathrm{H}_{2} \mathrm{SO}_{4} \quad$ by Erythromycin. Portugaliae Electrochimica Acta, 28(1), 1526, 2010.

[10] Mozaleva A., Mozaleval I., Hassel A., "The Voltage-Time Behaviour for Porous Anodizing of Aluminium in a FluorideContaining Oxalic Acid Electrolyte" Electrochemistry Communications, 3, 299305, 2002.

[11] Ebenso E., Okafor P., Eppe U., "Studies on the Inhibition of Al Corrosion by 2Acetylphenothiazine in Chloroacetic Acids," Anticorrosion, Methods and Materials, 50, 414-421, 2003.

[12] Blanc C., Gastaud S., Mankowski G., "Mechanistic Studies of the Corrosion of 2024 Aluminum Alloy in Nitrate Solutions," Journal of the Electrochemical Society, 150,396-404, 2003.

[13] Singh A., Quraishi M., "Study of Some Bidentate Schiff Bases of Isatin as Corrosion Inhibitors for Mild Steel in Hydrochloric Acid Solution", Int. J. Electrochem. Sci., 7, 3222-3241, 2012.

[14] Jores K., Mehnert W., Drecusler M., Bunyes H., Johan C., Mader K., "Investigation on the stricter of solid lipid nanopartuicles and oil-loaded solid nanoparticles by photon correlation spectroscopy, field flow fractionasition and transmission electron microscopy", J. Control Release. 17, 217- 227, 2014.

[15] Molpeceres J., Aberturas M., Guzman M., "Biodegradable nanoparticles as a delivery system for cyclosporine: preparation and characterization", J. Microencapsul. 17, 599-614. 2000.

[16] Eddy N. O, Ameh P. O., Gwarzo M. Y., Okopand I. J., Dodo S. N. "Physicochemical study and corrosion inhibition potential of Ficus tricopoda for aluminium in acidic medium. Portugaliae Electrochimica Acta”, 31(2), 79-93. 2013. 